\title{
BARRIERS AND STRATEGIES FOR THE CLINICAL TRANSLATION OF ADVANCED ORTHOPAEDIC TISSUE ENGINEERING PROTOCOLS
}

\author{
H. Madry ${ }^{1,2,6}$, M. Alini ${ }^{3,6}$, M.J. Stoddart ${ }^{3,6}$, C. Evans ${ }^{4}$, T. Miclau ${ }^{5}$ and S. Steiner ${ }^{3, *}$ \\ ${ }^{1}$ Center for Experimental Orthopaedics, Saarland University, Homburg, Germany \\ ${ }^{2}$ Department of Orthopaedic Surgery, Saarland University Medical Center, Homburg, Germany \\ ${ }^{3}$ AO Research Institute Davos, Davos, Switzerland \\ ${ }^{4}$ Center for Advanced Orthopaedic Studies, Beth Israel Deaconess Medical Center, \\ Harvard Medical School, Boston, MA, USA \\ ${ }^{5}$ Department of Orthopaedic Surgery, University of California at San Francisco/San Francisco General Hospital \\ Orthopaedic Trauma Institute, San Francisco, CA, USA \\ ${ }^{6}$ Collaborative Research Partner Acute Cartilage Injury Program of AO Foundation, Davos Platz, Switzerland
}

\begin{abstract}
Research in orthopaedic tissue engineering has intensified over the last decade and new protocols continue to emerge. The clinical translation of these new applications, however, remains associated with a number of obstacles. This report highlights the major issues that impede the clinical translation of advanced tissue engineering concepts, discusses strategies to overcome these barriers, and examines the need to increase incentives for translational strategies. The statements are based on presentations and discussions held at the AO Foundation-sponsored symposium "Where Science meets Clinics 2013" held at the Congress Center in Davos, Switzerland, in September, 2013. The event organisers convened a diverse group of over one hundred stakeholders involved in clinical translation of orthopaedic tissue engineering, including scientists, clinicians, healthcare industry professionals and regulatory agency representatives. A major point that emerged from the discussions was that there continues to be a critical need for early trans-disciplinary communication and collaboration in the development and execution of research approaches. Equally importantly was the need to address the shortage of sustained funding programs for multidisciplinary teams conducting translational research. Such detailed discussions between experts contribute towards the development of a roadmap to more successfully advance the clinical translation of novel tissue engineering concepts and ultimately improve patient care in orthopaedic and trauma surgery.
\end{abstract}

Keywords: Tissue engineering, translational barriers, clinical translation, regenerative medicine, articular cartilage repair.

*Address for correspondence:

Dr. Sandra Steiner

AO Research Institute Davos

Clavadelerstrasse 8

7270 Davos Platz, Switzerland

Telephone Number: 41814142452

FAX Number: 41814142299

Email: sandra.steiner@aofoundation.org

\section{Introduction}

In the past century, considerable advances have been made in the field of operative fracture management (Perren, 2008) and joint arthroplasty (Muller, 1992). Despite these advances, major problems, such as the repair of acute cartilage injuries, large bone defects, and annulus fibrosus ruptures, remain in the field of orthopaedic and trauma surgery and have been identified in previous AO Foundation workshops. While research in the field of regenerative medicine has intensified over the last decade and novel tissue engineering protocols continue to emerge, the translation of these new applications into the clinic remains a major hurdle for the realisation of new treatments. To address these barriers and to discuss strategies to overcome them, the AO Foundation sponsored the symposium "Where Science meets Clinics" that was held in Davos, Switzerland from September 5-7, 2013. The symposium organisers convened a diverse group of stakeholders involved in orthopaedic tissue engineering, including scientists, clinicians, health care industry professionals, and regulatory agency representatives.

The final half day of the symposium was dedicated to the discussion of the barriers impeding and strategies for advancing the clinical translation of promising tissue-engineering approaches. The session began with presentations from four keynote speakers, representing stakeholders from academia, the clinical arena, health care industry, or regulatory authority, who presented their views of the most significant barriers hampering the delivery of new technologies to the patient. These presentations were followed by a podium-led discussion that included all symposium participants to brainstorm on ways to overcome the major identified obstacles and incentivise clinical translational research.

This report highlights the key messages of the stakeholder presentations (Table 1) and includes the major conclusions from the subsequent podium discussions with the symposium participants. These conclusions contribute to the overarching discussion on what is needed to overcome existing barriers to translating research discoveries into clinical treatments. 
Table 1. Podium members "Barriers \& Strategies for Translation" session.

\begin{tabular}{|l|l|l|l|}
\hline \multicolumn{1}{|c|}{ Member } & \multicolumn{1}{c|}{ City } & \multicolumn{1}{c|}{ Country } & \multicolumn{1}{c|}{ Stakeholder } \\
\hline Lennart Akerblom & Uppsala & Sweden & Regulatory Agency \\
\hline Mats Brittberg & Kungsbacka & Sweden & Clinician \\
\hline Daniel Buchbinder & New York & USA & Moderator / Clinician \\
\hline Chris Evans & Boston & USA & Academic Scientist \\
\hline Jörg Goldhahn & Basel & Switzerland & Industry \\
\hline Henning Madry & Homburg & Germany & Clinician \\
\hline Christian Matula & Vienna & Austria & Clinician \\
\hline Anthony Ratcliffe & San Diego & USA & Industry \\
\hline Michael Schütz & Brisbane & Australia & Moderator / Clinician \\
\hline Norbert Südkamp & Freiburg i. Br. & Germany & Moderator / Clinician \\
\hline
\end{tabular}

\section{What are the Translational Barriers and How to Overcome Them?}

\section{An academic scientist's perspective}

In the first keynote lecture, Dr. Chris Evans gave insights into an academic biologist's perspective on research translation in the field of gene therapy and other biological modalities for orthopaedic conditions. He pointed out that biological protocols, such as those for gene therapy treatment, are much more difficult to translate than those for drugs or devices (Evans et al., 2012). Indeed, there is no gene therapy product yet available for an orthopaedic condition, although research is actively addressing gene-based therapies, particularly for the treatment of rheumatoid and osteo- arthritis (Evans et al., 2011), bone regeneration (Evans, 2010) and cartilage repair (Madry and Cucchiarini, 2013).

Among the non-scientific barriers, the multi-disciplinary nature of orthopaedic research, highly complex clinical conditions and the multifactorial nature of the translation process itself were viewed as major barriers for translation. Evans argued that the traditional linear model of research translation needs to be replaced by a supportive "translational research environment" where all components are in full and free communication from the beginning of the project.

Professional incentives, such as the tolerance for slower-to-develop transitional research programs with fewer resulting publications, are less than that for other basic research work, which can deliver faster results and more publications (Büchler et al., 2011). Further, funding these longer-term programs is more difficult with most of the current shorter-term research contracts and grants. This encourages many scientists to follow their natural disposition of continually refining basic research projects, rather than advancing findings along a translational track (Evans et al., 2011).

Moreover, the training and incentivisation of clinicianscientists, in particular with regards to Europe and North America, has become progressively difficult due to increasing financial constraints of universities and hospitals. Clinicians increasingly are required to generate more clinical income for their departments, and the decline in the number of clinician-scientists has already been addressed in several excellent review articles (Ahn et al., 2008a; Ahn et al., 2008b; Einhorn, 2006; Rosier, 2006).

At the same time, regulatory issues have become more complex, and there is no clear road map. Funding for purely translational work continues to be difficult, as often the emphasis of government research funding agencies is placed on basic research. Although industry supports clinical research, it is often difficult to commit companies to fund high-risk, innovative therapeutic approaches, especially over the long term. Moreover, there is a trend towards delaying support until a protocol has already shown evidence of success in the clinic.

Dr. Evans concluded that strategies for successful translation include the assembly of an interactive, critical mass of multidisciplinary experts interested in translating promising scientific findings, the creation of sustained funding for translational approaches, and the promotion of investigators through higher-degrees of academic independence.

The subsequent podium-led discussion supported these concepts. There was broad recognition that successful programmes require a strong working relationship between clinicians, scientists, and other stakeholders, and need to maintain a thorough understanding of the clinical condition. Finding and establishing collaborative multidisciplinary networks remain major challenges, and are complicated by the potentially differing needs of the stakeholders. Few financial incentives exist to motivate basic researchers into translational research careers, and the sustained support for the clinician-scientist is increasingly lacking. This suggests novel metrics for measuring academic performance may be needed. For many complex musculoskeletal diseases, the translation of new technologies into therapies is difficult, slower-to-develop, and underfunded. In order to better address these issues, the stakeholders will need to better educate the funding agencies, policy makers, general public and consumers, with the goal to develop policies that better reward and increase resources for translational research efforts. Additionally, collaborative interdisciplinary groups should continue to be supported to study disease-focused work such as that support provided by the AO Foundation's Collaborative Research Programs (CRPs). 


\section{A clinician's perspective}

Dr. Mats Brittberg, in his keynote talk "Cartilage repair; barriers and strategies for translation," pointed out several key issues negatively affecting translational research, including the academic culture and lack of clinicallyrelevant animal models. The academic culture, deeply rooted in the pursuit for basic science discoveries, often hinders the conduct of translational research activities; clinical trials are frequently not viewed as true academic excellence. Moreover, academic careers in clinical medicine are not as financially attractive, but translational research requires these physicians. Clinician input is not only required as a conduit from the bench to the patient, but is also essential in the development of disease-appropriate animal models.

In his work on articular cartilage, a tissue which has demonstrated poor regenerative capacity (Hunziker, 2009) and has few restorative therapies available (Brittberg, 2009), there is a clear lack of animal models that accurately mimic the human condition (Johnstone et al., 2013). For example, models that study the heterotopic implantation of cartilaginous constructs in subcutaneous pockets in nude mice have only remote relevance to a clinically apparent knee cartilage defect (Madry et al., 2013). For highly innovative, more clinically relevant cell-based techniques for cartilage repair, such as autologous chondrocyte implantation (ACI) (Brittberg et al., 1994), more complex models are required to study the open issues of optimal cell source and location, isolation and preparation of cells, requisite patient age, and the nature of the repair tissue (Johnstone et al., 2013).

Following the presentation, the discussion underscored the need for funding agencies to better recognise the importance of common debilitating musculoskeletal conditions. While these conditions may not be life threatening, they significantly influence society economically and patients' quality of life. Affected individuals and clinical practitioners often do not communicate with policy makers about their conditions, and thus research money is often directed to mortality-associated diseases. Solutions include improved advocacy efforts for musculoskeletal translational research and support for interdisciplinary clinical networks. Additionally, industry funding is essential for conducting clinical research studies, and ultimately for bringing novel therapies to the market. Therefore, the interdisciplinary teams need to include industry input early enough to help guide the work along pathways relevant to industry.

\section{An industry's perspective}

Dr. Anthony Ratcliffe provided a broad overview of the health care industry's perspective on research translation. To have a true clinical impact, potential products must be both therapeutically effective and commercially successful. A careful market assessment is essential for new products, and the inclusion of industry partners is important to maintain realistic direction for potential products. Ultimately, an industrial product manager oversees the entire production pathway, and the manufacturing process has to be validated and the American Society for Testing and Materials (ASTM) and the International Organisation for Standardisation (ISO) guidelines need to be followed. The technical data made available by industry collaborators can reduce possible future technical difficulties. Products should be developed for specific applications, rather than being developed without any clear use in mind. A high quality functional product design is important. Key preclinical studies that are tailored to the final product design and include essential issues, such as sterility or storage, need to be performed early during the development of a technology. Finally, industry can assist with the creation of an efficient manufacturing system and a robust business plan. For example, outsourcing, rather than "in-house" production may provide for greater budget flexibility and control, enhanced product-specific expertise, and reduced operating expenses. This is especially relevant for small companies.

Using articular cartilage repair as an example, the required devices are complex, and the product design must include successful mechanical properties, biological delivery, fixation of the device in situ, and generalisability to multiple joints. Successful products will need to be manufactured reproducibly in large quantities and be of high and consistent quality. A variety of tissue engineering products are already on the market, with 89 on-going articular cartilage repair trials, 20 of which involve cellbased therapies. The field is thus becoming crowded, raising concerns about market share and commercial success. The theoretical market size, addressing the older and active population, is projected to be 50-500 million USD (Communication from Anthony Ratcliffe and FDA estimates -http://www.fda.gov/downloads/ advisorycommittees/committeesmeetingmaterials/ b loodva c c i n e s a n d o the r biologi c s/ cellulartissueandgenetherapiesadvisorycommittee/ ucm170068.ppt).

The podium-based discussion emphasised the need to synergise communication between basic researchers, clinicians, and industry professionals, although individuals' needs, motivations, and research questions may differ. From an industry perspective, collaborations with academia can be very beneficial, particularly when collaborations involve multi-disciplinary teams of scientists and clinicians. Further, industry tends to acquire technologies when market opportunities outweigh the costs of the investments. There is a need to educate industry on evolving clinical treatments. The symposium attendees also recognised that the working opportunities for scientists in industry are increasingly perceived to be highly attractive.

\section{A regulatory agencies' perspective}

Dr. Lennart Åkerblom presented a talk entitled "Regulatory perspectives on the translation of chondrocyte implantation products". By definition [European Medicines Agency (EMA) and its committee for advanced therapies (CAT)], therapies that include gene therapy medicinal products, somatic cell therapy medicinal products and tissueengineered products - termed ATMP - are at the cutting edge of innovation and offer a major promise in the treatment of musculoskeletal diseases (Schneider et al., 
2010). When an ATMP is combined with one or more medical devices, such as scaffolds or implants, it is called "a combined ATMP".

The regulation of combined ATMPs is a very complex process, and approval for these products represents a major hurdle, as these products must satisfy both the medical device and ATMP pathways. A primary concern of the regulatory agencies is that the clinical benefits of any new technology needs to outweigh any potential health risks associated with a treatment. The role of a regulatory agency primarily is to protect patients, and not to bring new technologies to market as quickly as possible. With respect to clinical trials, a clearly defined disease indication, target population, and optimal disease stage for successful treatment are important considerations. Moreover, appropriate control populations and accurate clinical end-points need to be defined. From a regulatory agency's perspective, clinical effectiveness may or may not correlate with product potency. Potency assays need to be based on the intended biological effect, and potential risks that need to be evaluated include contamination, tumorigenicity, dedifferentiation, immunogenicity and ectopic engraftment.

Trials with preclinical large animal models are mandatory to obtain market approval, although it is clear that no animal model accurately mimics the human condition. In addition, multiple randomised controlled clinical trials (RCTs) are required, as the sole reliance on historical data will not satisfy the regulatory requirements. Extensive clinical trials represent a significant financial burden for the company developing the product. For example, for products containing chondrocytes (approximately 15,000 patients have been treated over the past 15 years), a 3-year follow up is necessary, although earlier time points are acceptable if histological and MRI data are available. Taken together, the extensive "package" of non-clinical, clinical, and published data required to obtain market approval of novel ATMPs requires much larger financial resources than the registration of a medical device. These requirements are often seen as hurdles that are extremely hard to overcome. It is possible that in the future, a more flexible regulatory approach based on evidence with high quality clinical data may emerge (specifically for ACI products), as some major companies remain reluctant to enter these markets due to the complex regulatory requirements. Indeed, the number of approved ATMPs is still relatively small.

Investigators frequently face a number of problems when dealing with regulatory agencies. Amongst the most significant of these issues is the absence of a clear road map for bringing tissue-engineered products to the market, which leads to the notion that the current regulatory pathway is too complex and difficult of a barrier to navigate. Other problems include: changing personnel at regulatory agency leading to shifting and non-binding opinions, lack of standards between different countries, and the limited ability to respond to the increased demands. All of these issues may negatively affect industry's interest to develop ATMPs. To reduce the complexity of these regulations and guidelines and to facilitate compliance, regulatory agencies should develop international standards and coordinate inter-agency regulatory agreements. This would greatly assist in removing some of the barriers.

From a regulatory perspective, agencies frequently are approached and engaged late in the product development process, which is not ideal. Investigators should approach their regulatory agencies early and often, and put a stronger focus on collaborating with these agencies when designing their studies. Investigators should summarise and document their meetings with their regulatory agencies and engage additional experts as needed.

\section{Conclusion}

The clinical translation of novel tissue engineering protocols and promising advanced therapies remains associated with a number of challenges. The major issues emerging from the discussions during this symposium include the need to improve the collaboration and communication between scientists, clinicians, industry and regulatory experts, the current lack of incentives for scientists to become involved in translation, the absence of a defined road map to the clinic, and the continued shortage of long-term funding for research teams involved in translational projects. Despite these barriers, continued progress in tissue engineering indicates that there are many reasons for continued optimism for highly innovative translational approaches.

To further address barriers to translational research, additional symposia and other similar activities that foster exchanges and networking between the diverse stakeholder groups are essential. Education of the public and policy leaders regarding the significant impact of musculoskeletal conditions is also paramount to improve the long-term funding opportunities. Finally, the multi-disciplinary stakeholders should engage the regulatory agencies early in the development of new technologies, as well as work with them when possible to create improved approval processes.

There is general consensus that a collaborative effort by all stakeholders involved in clinical translation is required to advance innovative tissue engineering therapies and ultimately to improve patient care in orthopaedic and trauma surgery.

\section{Acknowledgements}

The statements in this manuscript are based on presentations and podium-based discussions that took place during the session "Barriers \& Strategies for Translation" of the "Where Science meets Clinics" Symposium 2013, which was sponsored and organised by the AO Foundation in Davos, Switzerland. The authors thank the presenters, podium members, discussion moderators, and all the symposium participants for their valuable input and contributions. T. Miclau is a consultant for Amgen and is the chair of the Osteosynthesis and Trauma Care Foundation. He currently holds grants from Stryker and Synthes. The other authors have no conflict of interest. 
The abstracts from this meeting are available at: http://www.ecmjournal.org/journal/supplements/ vol026supp08/AO13.htm

\section{References}

Ahn J, Man LX, Wanderer J, Bernstein J, Iannotti JP (2008a) The future of the orthopaedic clinician-scientist. Part I: The potential role of MD-PhD students considering orthopaedic surgery. J Bone Joint Surg Am 90: 1794-1799.

Ahn J, Donegan DJ, Lawrence JT, Halpern SD, Mehta S (2008b) The future of the orthopaedic clinician-scientist. Part II: Identification of factors that may influence orthopaedic residents' intent to perform research. J Bone Joint Surg Am 92: 1041-1046.

Brittberg M (2009) ... more bricks to the building of cartilage knowledge? Knee Surg Sports Traumatol Arthrosc 17: $1275-1277$.

Brittberg M, Lindahl A, Nilsson A, Ohlsson C, Isaksson O, Peterson L (1994) Treatment of deep cartilage defects in the knee with autologous chondrocyte transplantation. N Engl J Med 331: 889-895.

Büchler MW, Diener MK, Weitz J (2011) Scientific evaluation of modern clinical research: we need a new currency! Langenbecks Arch Surg 396: 937-939.

Einhorn TA (2006) Funding the mandate for the orthopaedic clinician scientist. Clin Orthop Relat Res 449 : 76-80.

Evans $\mathrm{CH}$ (2010) Gene therapy for bone healing. Expert Rev Mol Med 12: e18.

Evans CH, Ghivizzani SC, Robbins PD (2011) Getting arthritis gene therapy into the clinic. Nat Rev Rheumatol 7: 244-249.

Evans CH, Ghivizzani SC, Robbins PD (2012) Orthopaedic gene therapy - lost in translation? J Cell Physiol 227: 416-420.

Hunziker EB (2009) The elusive path to cartilage regeneration. Adv Mater 21: 3419-3424.

Johnstone B, Alini M, Cucchiarini M, Dodge GR, Eglin D, Guilak F, Madry H, Mata A, Mauck RL, Semino CE, Stoddart MJ (2013) Tissue engineering for articular cartilage repair - the state of the art. Eur Cell Mater 25: 248-267.

Madry H, Cucchiarini M (2013) Advances and challenges in gene-based approaches for osteoarthritis. J Gene Med 15: 343-355.
Madry H, Rico AR, Venkatesan JK, Johnstone B, Cucchiarini M (2013) Transforming growth factor betareleasing scaffolds for cartilage tissue engineering. Tissue Eng Part B Rev 20: 106-125.

Muller ME (1992) Lessons of 30 years of total hip arthroplasty. Clin Orthop Relat Res 274: 12-21.

Perren SM (2008) Fracture healing. The evolution of our understanding. Acta Chir Orthop Traumatol Cech 75: 241-246.

Rosier RN (2006) Institutional barriers to the orthopaedic clinician-scientist. Clin Orthop Relat Res 449: 159-164.

Schneider CK, Salmikangas P, Jilma B, Flamion B, Todorova LR, Paphitou A, Haunerova I, Maimets T, Trouvin JH, Flory E, Tsiftsoglou A, Sarkadi B, Gudmundsson K, O’Donovan M, Migliaccio G, Ancans J, Maciulaitis R, Robert JL, Samuel A, Ovelgonne JH, Hystad M, Fal AM, Lima BS, Moraru AS, Turcani P, Zorec R, Ruiz S, Akerblom L, Narayanan G, Kent A, Bignami F, Dickson JG, Niederwieser D, Figuerola-Santos MA, Reischl IG, Beuneu C, Georgiev R, Vassiliou M, Pychova A, Clausen M, Methuen T, Lucas S, Schussler-Lenz M, Kokkas V, Buzas Z, MacAleenan N, Galli MC, Line A, Gulbinovic J, Berchem G, Fraczek M, Menezes-Ferreira M, Vilceanu N, Hrubisko M, Marinko P, Timon M, Cheng W, Crosbie GA, Meade N, di Paola ML, VandenDriessche T, Ljungman P, D’Apote L, Oliver-Diaz O, Buttel I, Celis $P$ (2010) Challenges with advanced therapy medicinal products and how to meet them. Nat Rev Drug Discov 9: 195-201.

\section{Discussion with Reviewers}

Reviewer I: Although not highlighted as a major obstacle by the authors, would an effort to equalise international standards be a worthwhile step to lowering some of the barriers?

Authors: We agree that to reduce the complexity of regulations and guidelines and to facilitate compliance, regulatory agencies should develop international standards and coordinate inter-agency regulatory agreements. This would greatly assist in removing some of the barriers. 